\title{
The Number of Triangulations on Planar Point Sets
}

\author{
Emo Welzl \\ Institute of Theoretical Computer Science \\ ETH Zurich, Switzerland
}

\begin{abstract}
We give a brief account of results concerning the number of triangulations on finite point sets in the plane, both for arbitrary sets and for specific sets such as the $n \times n$ integer lattice.
\end{abstract}

Given a finite point set $P$ in the plane, a geometric graph is a straight line embedded graph with vertex set $P$ where no segment realizing an edge contains points from $P$ other than its endpoints. We are interested in crossing-free geometric graphs on a given planar point set, i.e. segments are not allowed to share points other than common endpoints. A maximal crossing-free geometric graph on a point set $P$ is called a triangulation of $P$.
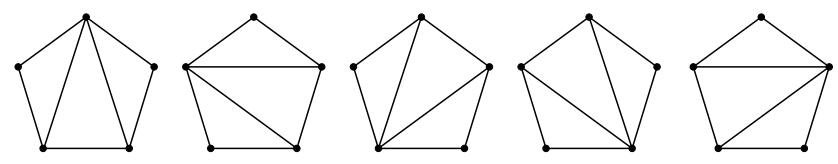

Fig. 1. All triangulations of five points in convex position

If, as it is the case in Fig. 1, the points are in convex position, i.e. vertices of a convex polygon, then every triangulation clearly must contain all edges of "its" convex polygon, and we are left with choosing a triangulation for this polygon. Euler was the first to consider how many choices there are for a convex $n$-gon, but it was proven only later that this number is $C_{n-2}$, where $C_{m}:=\frac{1}{m+1}\left(\begin{array}{c}2 m \\ m\end{array}\right)=$ $\Theta\left(m^{-3 / 2} 4^{m}\right)$, known as the Catalan numbers; (see, e.g., also Pólya's article $O n$ Picture-Writing [14]).

The example of four points already shows that position matters: Four points in convex position allow two triangulations, while there is only one otherwise. David Avis was perhaps the first to ask what the maximal possible number of triangulations of a general $n$-point set is. An upper bound of $n^{O(n)}$ is easy to obtain, but in 1982 Ajtai, Chvátal, Newborn, and Szemerédi [3] showed that for any set of $n$ points the number of all crossing-free geometric graphs is at most $c^{n}$ for $c=10^{13}$. The constant in the bound for triangulations has been successively improved [18/6 17/16]. The currently best bound of $43^{n}[15]$ is derived 
via considering random triangulations of finite point sets in general position with triangular convex hull. For a random non-extreme vertex in such a random triangulation one can show that it has degree 3 with probability at least $\frac{1}{43}$ ("random" refers here always to "uniformly at random"); interestingly, this yields the claimed bound on the number of triangulations.

Note that every crossing-free geometric graph is contained in some triangulation and a triangulation has at most $3 n-6$ edges. Therefore, any bound of the form $\tau^{n}$ for triangulations yields a bound of $2^{3 n-6} \tau^{n}<(8 \tau)^{n}$ for all crossing-free geometric graphs; so this stands at $344^{n}$.

The $43^{n}$-bound is probably far from optimal. On the other end [2] show that there are sets of $n$ points with as many as $\Omega\left(8.48^{n}\right)$ triangulations.

Lattice triangulations. The extremal properties for general point sets are still wide open, but even for "simple" concrete point sets the number of triangulations seems hard to analyze. One such example is the $n \times n$ integer lattice $L_{n \times n}:=$ $\{0,1, \ldots, n\}^{2}$ (with $(n+1)^{2}$ points); see [9] for a brief discussion of problems where lattice triangulations occur.

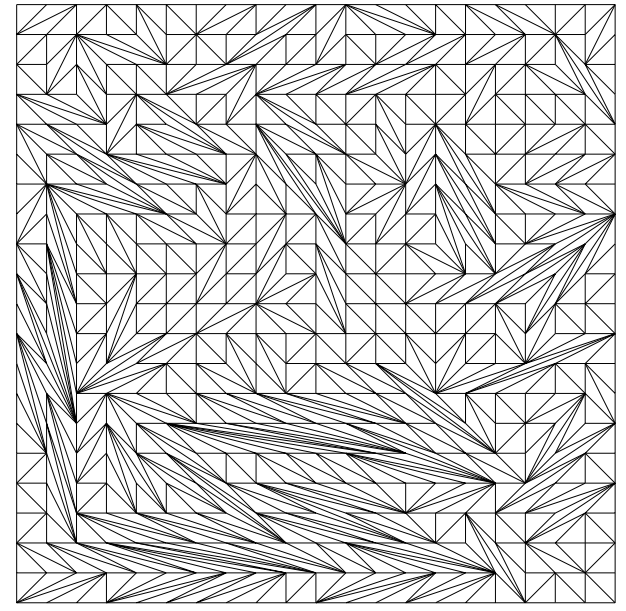

Fig. 2. Triangulated $20 \times 20$ lattice

The number of triangulations of $L_{n \times n}$ was first shown in 13 to be at most $64^{n^{2}}$ (this is, in fact, more than the general upper bound known today). Anclin [4] improved that to $8^{n^{2}}$ with the following argument: First, it is easy to show that in every triangulation each edge contains exactly one of the half-integral points $\left(\frac{1}{2}\{0,1, \ldots, 2 n\}\right)^{2} \backslash L_{n \times n}$ as its midpoint, and, conversely, every such halfintegral point lies on one of the edges. Second, he proves that if we choose the edges through these half-integral points in a row by row and left-to-right fashion, then at each point there are at most two choices compatible with the edges chosen so far. Half-integral points on the boundary leave no choice, so there are at most 
$3 n^{2}-2 n$ binary choices to be made which readily yields the $8^{n^{2}}$ bound. As one soon realizes, even interior half-integral points often allow only one choice as we get to them, which indicates that the bound over counts. Indeed, [10 argue that the bound can be set to $O\left(6.86^{n^{2}}\right)$, the best estimate currently known. The existence of at least $\Omega\left(4.15^{n^{2}}\right)$ triangulations on $L_{n \times n}$ was certified in [9].

Other special point sets. There are a few special types of configurations other than convex position for which the number of triangulations is known; see Fig. 3 for such sets (we skip formal definitions of the configurations and we ignore polynomial factors in the counting).
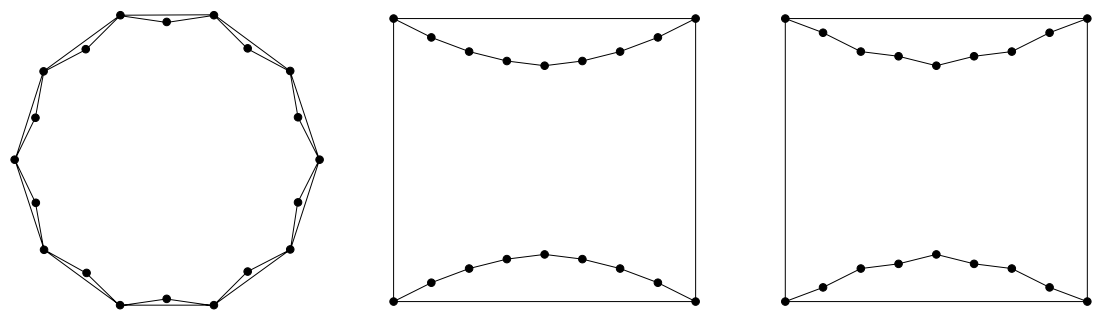

Fig. 3. The double circle (20 points), the double chain (18 points), and the double zig-zag chain (18 points). Edges shown are those which have to appear in every triangulation of the set.

The double circle has $\sqrt{12}^{n}, \sqrt{12} \approx 3.4641$, triangulations $[8$, which is obviously significantly less than for convex position and the smallest number known for $n$ points in general position (no three points on a line). For some time the double chain considered in [7] with $8^{n}$ triangulations was the set exhibiting the most triangulations known, but then 2] analyzed the double zig-zag chain with $\sqrt{72}^{n}, \sqrt{72} \approx 8.4853$, triangulations.

Algorithmic aspects. In [5] it is shown that the set of all triangulations of a point set can be enumerated in time $O(t \cdot \operatorname{poly}(n))$, where $t$ is the number of triangulations. But when it comes to counting, i.e. computing this number $t$, nothing is known at all other than some heuristics [1].

An interesting related question is that of the mixing rate of the random walk on the flip graph of all triangulations of a given point set. In this graph the triangulations represent vertices, and two triangulations are adjacent if we can obtain one from the other by removing one edge and replacing it by another one (an operation called edge-flip). This graph is connected and has diameter $O\left(n^{2}\right)$. A random walk (with some waiting time for technical reasons) will eventually produce a random triangulation, but nothing is known about the time it takes for that to happen - other than the case of points in convex position, where polynomial time mixing has been demonstrated [1112. Polynomial time mixing of this random walk would imply polynomial time generation of an approximate 
random triangulations, and (with help of the result in [15]) polynomial time approximate counting of triangulations.

\section{References}

1. O. Aichholzer, The path of a triangulation, Proc. 15th Ann. ACM Symp. on Computational Geometry (1999), 14-23.

2. O. Aichholzer, T. Hackl, H. Krasser, C. Huemer, F. Hurtado, and B. Vogtenhuber, On the number of plane graphs, Proc. 17th Ann. ACM-SIAM Symp. on Discrete Algorithms (2006), 504-513.

3. M. Ajtai, V. Chvátal, M. M. Newborn, and E. Szemerédi, Crossing-free subgraphs, Annals Discrete Math. 12 (1982), 9-12.

4. E. E. Anclin, An upper bound for the number of planar lattice triangulations, $J$. Combinat. Theory, Ser. A 103 (2003), 383-386.

5. D. Avis and K. Fukuda, Reverse search for enumeration, Discrete Appl. Math. 65 (1996), 21-46.

6. M. O. Denny and C. A. Sohler, Encoding a triangulation as a permutation of its point set, Proc. 9th Canadian Conf. on Computational Geometry (1997), 39-43.

7. A. García, M. Noy, and J. Tejel, Lower bounds on the number of crossing-free subgraphs of $K_{N}$, Comput. Geom. Theory Appl. 16 (2000), 211-221.

8. F. Hurtado and M. Noy, Counting triangulations of almost-convex polygons, Ars Combinatorica 45 (1997), 169-179.

9. V. Kaibel and G. Ziegler, Counting lattice triangulations, British Combinatorial Surveys (C.D. Wensley, ed.), Cambridge University Press, 2003.

10. J. Matoušek, P. Valtr, and E. Welzl, On two encodings of lattice triangulations, manuscript (2006).

11. L. McShine and P. Tetali, On the mixing time of the triangulation walk and other Catalan structures, in: DIMACS-AMS volume on Randomization Methods in Algorithm Design (Eds. P.M. Pardalos, S. Rajasekaran, and J. Rolim) DIMACS Series in Discrete Mathematics and Theoretical Computer Science 43 (1998), 147-160.

12. M. Molloy, B. Reed, and W. Steiger, On the mixing rate of the triangulation walk, in: DIMACS-AMS volume on Randomization Methods in Algorithm Design (Eds. P.M. Pardalos, S. Rajasekaran, and J. Rolim), DIMACS Series in Discrete Mathematics and Theoretical Computer Science 43 (1998),179-190.

13. S. Yu. Orevkov, Asymptotic number of triangulations in $\mathbf{Z}^{2}$, J. Combinat. Theory, Ser. A 86 (1999), 200-203.

14. G. Pólya, On picture-writing, The American Mathematical Monthly 63 (1956), 689-697. Also in: G. L. Alexanderson, The Random Walks of George Pólya, MAA, 2000.

15. M. Sharir, E. Welzl, Random triangulations of planar point sets, Proc. 22nd Ann. ACM Symp. on Computational Geometry (2006), 273-281.

16. F. Santos and R. Seidel, A better upper bound on the number of triangulations of a planar point set, J. Combinat. Theory, Ser. A 102 (2003), 186-193.

17. R. Seidel, On the number of triangulations of planar point sets, Combinatorica 18 (1998), 297-299.

18. W.S. Smith, Studies in Computational Geometry Motivated by Mesh Generation, Ph. D. Thesis, Princeton University, 1989. 